Documentation et bibliothèques

\title{
La coopération du Canada avec l'Afrique francophone dans le domaine des bibliothèques et de la documentation
}

\section{Rosemary Murray-Lachapelle}

Volume 25, numéro 2, juin 1979

URI : https://id.erudit.org/iderudit/1054359ar

DOI : https://doi.org/10.7202/1054359ar

Aller au sommaire du numéro

\section{Éditeur(s)}

Association pour l'avancement des sciences et des techniques de la documentation (ASTED)

\section{ISSN}

0315-2340 (imprimé)

2291-8949 (numérique)

Découvrir la revue

\section{Citer cet article}

Murray-Lachapelle, R. (1979). La coopération du Canada avec l'Afrique francophone dans le domaine des bibliothèques et de la documentation. Documentation et bibliothèques, 25(2), 81-86. https://doi.org/10.7202/1054359ar
Résumé de l'article

Les bibliothèques de l'Afrique francophone font face à de multiples problèmes. En nombre insuffisant, elles n'ont que des collections restreintes et manquent de personnel. Les bibliothèques des secteurs scolaires et publics, en particulier, ont été négligées. Face à ces besoins, l'ACDI (Agence canadienne de développement international) et spécialement le CRDI (Centre de recherches pour le développement international) ont mis sur pied, en Afrique francophone, des projets d'établissement de bibliothèques et de centres de documentation. La contribution canadienne reste toutefois minime. Le Canada n'a toujours pas de politique de coopération en matière de bibliothèque avec les pays en voie de développement, bien que certaines structures soient déjà en place. Aussi les bibliothécaires devraient-ils unir leurs efforts à ceux des autres spécialistes de l'information afin de sensibiliser davantage le gouvernement à l’importance de l’information dans le processus du développement.
Tous droits réservés (C) Association pour l'avancement des sciences et des techniques de la documentation (ASTED), 1979
Ce document est protégé par la loi sur le droit d'auteur. L’utilisation des services d'Érudit (y compris la reproduction) est assujettie à sa politique d'utilisation que vous pouvez consulter en ligne.

https://apropos.erudit.org/fr/usagers/politique-dutilisation/ 


\title{
La coopération du Canada avec l'Afrique francophone dans le domaine des bibliothèques et de la documentation
}

\author{
Rosemary Murray-Lachapelle \\ Bibliothèque du Parlement \\ Ottawa
}

Les bibliothèques de l'Afrique francophone font face à de multiples problèmes. En nombre insuffisant, elles n'ont que des collections restreintes et manquent de personnel. Les bibliothèques des secteurs scolaires et publics, en particulier, ont été négligées. Face à ces besoins, l'ACDI (Agence canadienne de développement international) et spécialement le CRDI (Centre de recherches pour le développement international) ont mis sur pied, en Afrique francophone, des projets d'établissement de bibliothèques et de centres de documentation. La contribution canadienne reste toutefois minime. Le Canada n'a toujours pas de politique de coopération en matière de bibliothèque avec les pays en voie de développement, bien que certaines structures soient déjà en place. Aussi les bibliothécaires devraient-ils unir leurs efforts à ceux des autres spécialistes de l'information afin de sensibiliser davantage le gouvernement à l'importance de l'information dans le processus du développement.

The libraries of francophone Africa have to deal with multiple problems. Insufficient in number, they have limited collections and a shortage of personnel. School and public libraries have been particularly neglected. Faced with these needs, CIDA (Canadian International Development Agency) and especially the IDRC (International Development Research Centre) have started some projects for the creation of libraries and documentation centers in that area. However, the Canadian contribution remains small. Although some structures are already in place, Canada still does not have a policy of cooperation in the field of libraries in developing countries. Also, the librarians should join their efforts to those of other information specialists in order to convince the government of the importance of information in the process of development.

Las bibliotecas de los países de habla francesa de África se enfrentan con varios problemas. Su número es insuficiente, sus colecciones están limitadas y les falta personal. Las bibliotecas de los sectores escolares y públicos han sido particularmente ignorados. Frente a estas necesidades, la ACDI (Agence canadienne de développement international) y sobre todo el CRDI (Centre de recherches pour le développement international) han creado proyectos para establecer bibliotecas y centros de documentación en los países de habla francesa de África. Sin embargo, la participación canadiense sigue siendo mínima. El Canadá todavia no tiene, en materia de bibliotecas, una política de cooperación con los países en vía de desarrollo, aunque existen ciertas estructuras. Así, los bibliotecarios deberian unir sus esfuerzos a los de otros especialistas de la información para convencer el gobierno de la importancia de la información en el proceso de desarrollo.

Les bibliothèques de l'Afrique francophone font face à plusieurs problèmes dont le plus important tient à leur nombre insuffisant. Dans un pays comme le Sénégal par exemple, une bibliothèque universitaire dessert une population de $4,885,000$ $(1975)^{1}$. Les bibliothèques nationales sont

1. Unesco, Annuaire statistique, 1976, Paris, 1977, $1.1,10.1$. 
rares et en conséquence le contrôle bibliographique demeure fort limité.

La plupart des bibliothèques n'ont pu développer que des collections inadéquates en raison du prix d'achat élevé des documents. Comme ailleurs, la majorité de ceux-ci sont importés d'Europe ou d'Amérique du Nord, des frais très élevés de transport viennent se greffer sur des prix déjà exorbitants. Il en résulte une restriction générale des politiques de prêt obligeant les usagers à consulter les collections sur place.

Les bibliothèques font aussi face à une grave pénurie de personnel qualifié. II n'existe, dans toute l'Afrique francophone de l'Ouest, qu'un seul centre de formation professionnelle, soit l'École des bibliothécaires, archivistes et documentalistes (E.B.A.D.) de Dakar.

On constate malgré tout, dans le secteur des bibliothèques scientifiques et techniques dont l'importance pour le développement socio-économique à l'échelle du continent est de plus en plus nettement perçue, des progrès encourageants, la plupart de ces centres restant toutefois le privilège des zones urbaines à forte densité de population. Dans les zones rurales d'accès difficile, où l'analphabétisme est très répandu, les bibliothèques municipales et scolaires demeurent quasi inexistantes et ne peuvent satisfaire le besoin d'ouvrages en langue vernaculaire auquel les publications étrangères ne peuvent guère répondre ${ }^{2}$.

Afin de résoudre ces problèmes et de faire face à ces besoins, l'Agence canadienne de développement international (ACDI) et, plus récemment, le Centre de recherches pour le développement international (CRDI) ont élaboré divers projets d'établissement de bibliothèques en Afrique francophone.

C'est en 1968 que le Bureau de l'aide extérieure, chargé depuis 1950 d'administrer les programmes «d'aide» du Canada, devenait l'Agence canadienne de déve-

2. Pour une étude plus détaillée de la situation des bibliothèques en Afrique, le lecteur peut se référer à Jean de Chantal, «Bibliothèques et archives du Tiers-Monde: problèmes et perspectives", Documentation et bibliothèques, vol. 21, no 2 (juin 1975), 85-95. loppement international ${ }^{3}$. Responsable au Gouvernement de l'élaboration et de l'exécution de la politique canadienne en matière de développement international, l'ACDI s'occupe des programmes d'assistance technique, c'est-à-dire de «l'envoi d'experts, d'enseignants, de conseillers dans les pays en voie de développement et de la venue au Canada ou dans un pays du Tiers-Monde d'étudiants et de stagiaires. " ${ }^{4}$ C'est surtout dans le cadre des programmes d'assistance technique que l'ACDI a parrainé les projets de développement de bibliothèques en Afrique francophone.

Au cours des dix dernières années, près d'une vingtaine de bibliothécaires ont fait, sous les auspices de l'ACDI, des stages en Afrique francophone: un en Algérie, deux au Bénin, deux au Cameroun, un au Gabon, un à l'île Maurice ${ }^{5}$, deux en Côte d'Ivoire, deux en Mauritanie, deux au Rwanda, deux au Sénégal et deux en Tunisie $^{6}$. Ces bibliothécaires comptaient

3. Louis Sabourin, «Analyse des politiques de coopération internationale du Canada», in Paul Painchaud, Le Canada et le Québec sur la scène internationale, Montréal, Presses de l'Université du Québec, 1977, p. 227.

4. Ibid., p. 240.

5. Même si sa langue officielle est l'anglais, l'île Maurice a été, pour les fins de cet article, assimilée aux pays de l'Afrique francophone, puisque le français parlé et lu y est d'un usage répandu, le créole restant la langue populaire.

6. Des statistiques plus précises sont difficiles à obtenir parce que l'accès aux dossiers par discipline n'existe pas pour les années antérieures à 1976. A partir de 1976 les dossiers portant sur les ressources humaines ont été intégrés au système informatique rendant les données par discipline immédiatement disponibles depuis lors. L'ACDI a envoyé depuis 1976 six bibliothécaires en Afrique francophone. Ces chiffres cependant ne comprennent pas les bibliothécaires travaillant dans le cadre d'un projet réalisé au nom de l'ACDI par une agence extérieure, une université par exemple. Les statistiques utilisées ici ont été tirées d'entrevues avec divers directeurs de projet de I'ACDI, d'échanges avec Jean de Chantal, bibliothécaire en chef au CRDI, de rapports personnels de l'auteur avec certains projets et des sources bibliographiques suivantes: F.W. Matthews et Doreen Fraser, Directory of Persons in Canada with Overseas Experience in Library and Information Services, Ottawa, International Development Research Centre, 1976, 16 p., et Sheila Summerhays, Programmes of the Canadian International Development Agency and the British Council in Assisting Library Development Abroad, Toronto, Faculty of Library Science, University of Toronto, 1972, $32 \mathrm{p}$. 
parmi leurs responsabilités le catalogage, les activités de référence, la compilation de la bibliographie nationale, la formation du personnel ainsi que l'organisation, le développement et l'administration des bibliothèques et de leurs ressources.

Deux projets ont particulièrement retenu I'attention de I'ACDI: la planification et la construction de la Bibliothèque nationale de la Côte d'Ivoire à Abidjan et l'organisation de la bibliothèque universitaire de l'île Maurice.

La fondation de la Bibliothèque nationale de la Côte d'Ivoire visait à créer une agenće centrale responsable du développement des bibliothèques et du contrôle bibliographique à travers le pays. Mme K.L. Liguer-Laubouet, la bibliothécaire nationale, en définit le rôle comme suit: 1) planifier et organiser le développement des bibliothèques (scolaires, municipales, spécialisées et universitaires); 2) superviser le fonctionnement de ces établissements et centraliser l'étude des problèmes techniques et professionnels les concernant; 3) étudier et proposer une législation appropriée à ces établissements; 4) assurer la promotion du livre et favoriser le développement de l'édition en Côte d'Ivoire?.

Afin de remplir ce vaste mandat, on y avait prévu une bibliothèque modèle pour enfants, une collection de documents audiovisuels, une librairie, un amphithéâtre de 120 sièges et un café en plein air. Les étudiants, tant au niveau primaire que secondaire et universitaire, tout comme le grand public, jouiraient des privilèges d'emprunt et des autres services ${ }^{8}$.

De 1968 à 1974, époque de l'achèvement du projet, l'ACDI a dépensé \$1.5 millions pour l'exécution des travaux. La firme montréalaise Longpré, Marchand, Goudreau, Dobush, Stewart, Bourke Architectes en conçut le plan et réalisa la construction, un bibliothécaire canadien

7. "La Bibliothèque nationale de Côte d'lvoire", Jeune Afrique, no 627 (13 janvier 1973), 35.

8. Madeleine Vaillancourt Wagner, «Urgence-bibliothèques en Afrique: deux approches complémentaires en Côte d'Ivoire et à l'île Maurice». Coopération Canada, no 25 (1976), 17. assumant les fonctions techniques à titre de conseiller pour le compte de l'ACDI ${ }^{9}$. Un second bibliothécaire chargé de rassembler autant que possible les éléments d'une bibliographie nationale fut envoyé plus tard sur le terrain.

Le projet, surtout à cause des sommes considérables qui y furent consacrées à un moment où il aurait pu sembler préférable de satisfaire des besoins plus élémentaires $^{10}$, a fait l'objet de critiques sévères qu'il faudrait cependant pondérer à la lumière de la politique générale de développement des bibliothèques à travers l'Afrique francophone ${ }^{11}$.

De 1970 à 1974, l'ACDI prit également des engagements envers la bibliothèque de l'Université de l'île Maurice. Le but de ce projet était d'organiser et de développer les ressources de la bibliothèque universitaire. C'est dans ce cadre que l'ACDI envoya un Canadien à titre de bibliothécaire conseiller en chef, contribua à financer l'achat d'équipements et défraya pour deux étudiants le coût d'une bourse d'étude en bibliothéconomie. Ces derniers, après avoir complété leur formation, sont retournés à l'île Maurice, l'un à titre de directeur de la bibliothèque universitaire, l'autre comme directeur de la bibliothèque de l'Institut mauricien d'éducation.

Le Canada a ainsi parrainé la formation en bibliothéconomie de dix-sept étudiants d'Afrique francophone: la plupart ont été dirigés vers l'Université de Montréal, un vers l'Université d'Ottawa et un autre vers les bibliothèques du Québec et de l'Ontario pour un stage d'un an.

Comme on peut le constater, les projets touchant les secteurs documentaires et les bibliothèques n'absorbent qu'une très faible partie des ressources de l'ACDI. Ceci semble particulièrement évident si l'on regarde ses effectifs outre-mer. En 1975 par exemple, 1,500 conseillers, coopérants ou

9. Pour un examen des concepts d'architecture, voir Hélène Gosselin Geoffrion, «Abidjan, Bibliothèque nationale», Architecture concept, vol. 31, no 334 (mars-avril 1976), 14-18.

10. Madeleine Vaillancourt Wagner, “Urgence-bibliothèques...", 17.

11. Jean de Chantal, «Bibliothèques et archives du Tiers-Monde...». 85-95. 
«experts» travaillaient sur le terrain, en plus des quatre-vingts représentants affectés aux ambassades canadiennes à l'étranger $^{12}$. C'est donc surtout grâce aux travaux du Centre de recherches pour le développement international que s'élargit considérablement la contribution canadienne aux bibliothèques de l'Afrique francophone.

Établi en 1970 par une loi du Parlement, le CRDI reçut comme mission " d'entreprendre, d'encourager, de soutenir et de poursuivre des recherches sur les problèmes des régions du monde en voie de développement et sur les moyens d'application et d'adaptation des connaissances scientifiques, techniques, et autres au progrès économique et social de ces régions. »13

L'une des quatre divisions du CRDI, celle des Sciences de l'information, a énoncé en ces termes l'esprit qui l'anime:

«... n'existe-t-il pas encore une prise de conscience suffisante de l'importance de ce sujet (sciences de l'information) pour le développement économique et social. Même les pays industrialisés n'ont pas encore prévu les infrastructures adéquates, tant du point de vue capital humain que ressources, pour assurer à l'être humain les systèmes d'information dont il a besoin pour lui éviter d'épuiser ses forces à redécouvrir et à reconstruire ce qui en fait est déjà connu et établi.»14

Cette orientation du CRDI l'a poussé à mettre sur pied et à supporter une grande variété d'activités en sciences de l'information. Dès sa fondation, en coopération avec le département des Affaires économiques et sociales des Nations Unies, l'Unesco, le Bureau international du travail et l'OCDE, le Centre a participé activement à l'élaboration du Devsis (Système d'information sur

12. Louis Sabourin, “Analyse des politiques...", p. 228.

13. Centre de recherches pour le développement international, Les premiers 200 projets, Ottawa, 1974, $39 \mathrm{p}$

14. "La Division des Sciences de l'information», Le CRDI informe, vol. 2, no 1 (mars 1973), 3. les sciences du développement) ${ }^{15}$. Le Centre a aussi promu la recherche dans divers secteurs en difficulté tels que les besoins communautaires en services de bibliothèque, les conditions présentes de formation des bibliothécaires en Afrique ainsi que le contrôle bibliographique des documents africains, officiels et autres. En relation avec ces problèmes, le Centre a subventionné des ateliers de travail et de recherche réunissant les bibliothécaires africains. II parraine un projet de cueillette de données pour faciliter l'établissement de cartes thématiques de l'Afrique de l'Ouest, ainsi qu'un projet d'expansion d'un centre d'information des sols en République Centrafricaine. En dernier lieu, le Centre, dont le programme de publications est considérable, apporte son aide financière à certaines initiatives touchant des publications africaines, par exemple le numéro spécial de Blibad: bulletin de liaison à l'intention des bibliothécaires, archivistes et documentalistes africains (octobre 1977); de plus, la revue Famille et développement doit sa parution continue à l'appui apporté par le CRDI ${ }^{16}$.

Malgré toutes ces activités, le CRDI, outre son personnel employé au siège social à Ottawa ou dans les bureaux régionaux de l'Afrique, de l'Asie et de l'Amérique latine, ne mobilise sur place qu'un minimum d'effectifs canadiens. Cela s'explique d'une part par le fait que le CRDI, en tant que centre de recherche, ne s'intéresse pas à l'assistance technique, mais surtout par sa politique qui s'énonce ainsi:

«Nous nous opposons toujours à la formule d'aide courante qui consiste à déléguer une main-d'oeuvre étrangère, du fait que cette formule produit souvent l'effet contraire de celui escompté surtout lorsqu'il est jugé que

15. Devsis: esquisse d'un système international d'information pour les sciences du développement, Ottawa, Centre de recherches pour le développement international, 1976, $249 \mathrm{p}$.

16. Pour mieux connaître les activités du CRDI dans ce secteur, se référer aux sources suivantes: «La Division...», 16 p.; Centre de recherches pour le développement international, «Les premiers 200...». 39 p.; Centre de recherches pour le développement international, Projets 1975Ottawa, 1975- 
cette formule va à l'encontre des intérêts durables des pays qu'elle est censée servir." ${ }^{17}$

Dans un cas cependant, le Centre s'est employé à réaliser un projet sur le terrain, ce qui normalement relève de l'ACDI. "I s'agit d'un programme de formation de techniciens-animateurs à l'île Maurice, projet-pilote financé par le CRDI $(\$ 94,700$.) et utilisant des méthodes expérimentales.

Pour desservir une population d'un million d'habitants, l'île Maurice ne compte au total que huit bibliothécaires dont quatre au service de la bibliothèque universitaire. Les bibliothécaires en place, auxquels s'ajoute le directeur de programme venu de l'étranger, offrent, à l'université même, un programme d'études de quinze mois destiné à préparer des effectifs mauriciens en bibliotechnique. De plus, par une voie nouvelle et dynamique ajoutée au programme, les techniciens, gradués d'écoles secondaires et déjà employés dans les bibliothèques publiques et scolaires, peuvent recevoir une formation non seulement en administration des bibliothèques mais aussi en animation communautaire et diffusion de l'information. Dans le cadre d'un programme de développement rural financé par la Banque mondiale, les futurs diplômés devront travailler avec les enseignants à combattre l'analphabétisme, coopérer avec les travailleurs sociaux à la cueillette d'informations sur la nutrition et l'hygiène, etc. II est encore trop tôt pour porter un jugement sur un tel projet car ces premiers diplômés, au nombre de vingt, ne seront reçus qu'au printemps 1979. Plusieurs autres pays africains manifestent néanmoins un intérêt de plus en plus grand pour ce type de programme ${ }^{18}$.

Une activité plus typique du CRDI est signalée dans le rapport Projet de réseau d'information et de documentation scientifiques et techniques pour le Sahel; étude d'une stratégie documentaire en vue de la

17. "La Division...». 13.

18. Madeleine Vaillancourt Wagner, «Urgence-bibliothèques...», 25 création d'un tel réseau pour le CILSS à I'Institut du Sahel'", (Ottawa, CRDI, 1978) ${ }^{19}$.

C'est le Comité permanent inter-états de lutte contre la sécheresse dans le Sahel (CILSS) qui avait pris l'initiative d'une recherche à ce sujet, pour donner suite à la demande officielle présentée par les étatsmembres de l'Afrique de l'Ouest. Le rapport souligne «l'impérieuse nécessité de pouvoir disposer, à tout moment, des informations les plus récentes et les plus complètes... ${ }^{20}$, et définit les objectifs visés par l'opération: "l'autosuffisance alimentaire, l'organisation du développement rural, la lutte contre la désertification et la mise en oeuvre de techniques modernes, adaptées aux conditions du milieu. ${ }^{21}$ II évalue aussi les ressources actuelles dans le Sahel et identifie le groupe d'usagers susceptibles d'en tirer profit. II définit enfin la nature des besoins en termes de services d'information, de personnel, de méthodes et de formation pour développer les ressources en un réseau sahélien. Notons les recommandations suivantes: que chaque état-membre 1) définisse une politique nationale en matière de documentation; 2) établisse des normes et des classifications pour le personnel de bibliothèque; 3 ) organise des programmes de formation intermédiaire complémentaire à ceux offerts par l'E.B.A.D. à Dakar; 4) finalement, on y exprime le souhait que ces efforts soient coordonnés entre les étatsmembres. Le rapport présente en terminant un avant-projet d'ensemble signalant, entre autres, les sources de financement et la possibilité de recours, pour une période limitée, à l'assistance technique.

Le rapport revêt un grand intérêt pour deux raisons: d'abord parce qu'il propose une stratégie documentaire, et parce qu'il reconnaît la possibilité d'organiser les ressources existantes par des moyens traditionnels antérieurement ou simultanément

19. Djiby Sall et Maurice D. Cetherinet, Projet de réseau d'information et de documentation scientifiques et techniques pour le Sahel; étude d'une stratégie documentaire en vue de la création d'un tel réseau pour le CILSS à I'Institut du Sahel, Ottawa, Centre de recherches pour le développement international, 1978, $78 \mathrm{p}$.

20. Ibid., p. 6

21. Ibid., p. 3. 
à l'utilisation du processus d'informatisation, lequel est très cher et complexe tant sur le plan de l'entretien que de la technique. Aussi longtemps que les moyens traditionnels choisis demeurent compatibles avec une intégration ultérieure à un système informatique, le projet paraît souhaitable et réalisable.

Ces initiatives n'ont pas été les seules dans le domaine des bibliothèques et de la documentation. Le SUCO (Service universitaire canadien outre-mer), par exemple, a envoyé à l'occasion des bibliothécaires en Afrique francophone; le Centre du livre outre-mer a produit du matériel scolaire en langue vernaculaire spécialement conçu à l'intention des niveaux primaire et secondaire. Ces quelques réalisations d'organisations non gouvernementales, de même que les programmes d'associations comme celui de la participation canadienne aux activités de l'IFLA (Fédération internationale des associations de bibliothécaires) et le réseau à l'étranger des centres de documentation du ministère des Affaires extérieures pourraient fournir matière à une nouvelle recherche.

Les faits qui viennent d'être rapportés démontrent bien que le Canada n'a pas de politique de coopération définie, en Afrique francophone ou ailleurs, dans les secteurs des bibliothèques et de la documentation, en dépit des besoins essentiels dans ce domaine. Toutefois, les mécanismes pour l'adoption d'une telle politique existent.

Le document explicitant la stratégie de I'ACDI publié en 1975 met en lumière la notion de "self-reliance", reconnaît la nécessité des progrès à faire en matière d'éducation de base et de formation et exprime son appui aux centres de recherche et d'innovation ${ }^{22}$. De même, le CRDI conçoit les sciences de l'information comme un outil nécessaire à la «self-reliance»; le projet sahélien du CRDI fournit un bel exemple de stratégie documentaire répondant à un besoin pressant. Comment

22. Canada: stratégie de coopération au développement international 1975-1980, Ottawa, Agence canadienne de développement international, $1975,53 \mathrm{p}$. se fait-il alors que les bibliothèques ne se voient pas accorder une plus grande priorité dans les politiques globales de développement? La raison semble être d'ordre politique.

Selon les auteurs d'un article récent qui réclament une stratégie pour les systèmes internationaux d'information, le principal problème est politique. "The crux of the problem is how to impress on decisionmakers in government the importance of information.» ${ }^{23} \mathrm{Au}$ mois de juin 1979, un séminaire organisé conjointement par I'ASTED (Association pour l'avancement des sciences et des techniques de la documentation) et la CLA (Canadian Library Association) abordera cette question. Ce sera une première étape - importante - de franchie.

Notre point de vue est partagé par d'autres, comme l'indique la déclaration de la Table ronde Nord-Sud de mai 1978, organisée par la Société pour le développement international:

"Malheureusement, le dialogue NordSud a été entravé dans sa progression par l'insuffisance des systèmes internationaux d'information existant actuellement... Nous sommes amenés à penser qu'une restructuration fondamentale des systèmes d'information internationaux actuels représenterait un élément essentiel du nouvel ordre économique international. ${ }^{24}$

L'auteur suggère donc que les bibliothécaires dépassent les frontières de leur propre profession et unissent leurs efforts à ceux des autres spécialistes de l'information (scientistes de l'information, journalistes, archivistes, animateurs et éditeurs) afin de sensibiliser le gouvernement au rôle essentiel qu'ils doivent jouer dans le processus du développement.

23. Elizabeth K. Miller and Kate Wild, «A strategy for international information systems", Special Libraries, vol. 64, no. 11 (November 1978), 441.

24. Société internationale pour le développement, Table ronde Nord-Sud. Première session, Rome, 18-20 mai 1978, "Déclaration», Revue du développement international, vol. 20, no 2 (1978), 40. 